\title{
Amblyomma chabaudi Rageau, 1964 (Ixodidae)
}

\author{
Elevage au laboratoire. Description de la larve. \\ Observations complémentaires sur la nymphe.
}

\author{
Par G. UILENBERG
}

\begin{abstract}
Le mâle d' $A$. chabaudi a été décrit par Rageau (1964), la femelle et la nymphe par Uilenberg (1965).

Nous avons eu l'occasion d'élever cette espèce au laboratoire, à partir d'une femelle presque gorgée, récoltée sur une Pyxis arachnoides, par M. P. Malzy, zoologiste à l'Institut de Recherche Scientifique de Madagascar, qui a également fourni des tortues des espèces Pyxis arachnoides, Testudo radiata et Testudo planicauda.

Nous donnons ci-dessous les observations faites sur le cycle biologique d' $A$. chabaudi, la description de la larve, stade qui n'était pas encore connu, et nous complétons la description de la nymphe, dont jusqu'ici un seul spécimen (gorgé) avait été trouvé.
\end{abstract}

\section{Elevage d'Amblyomma chabaudi}

\section{Méthode :}

Les stades non parasitaires ont été gardés à l'étuve à tiques à une température de 27 à $28^{\circ} \mathrm{C}$ et à une humidité relative d'environ $90 \%$. L'élevage a été effectué dans cette même étuve sur les tortues et sur un lapin et à la température et l'humidité ambiantes du laboratoire sur deux autres lapins (moyenne d'environ $20^{\circ}$ et 60 à $70 \%$ d'humidité). La technique de l'élevage sur tortue était simple : l'animal était placé dans un grand cristallisoir couvert d'une toile; il restait à jeun pendant toute la période d'infestation afin de garder le cristallisoir propre (aucune déjection après le $4^{\circ}$ jour). Pour élever les tiques sur les oreilles de lapin, la méthode de Bailey (1960) a été suivie. 
Durée des différentes périodes du cycle:

(Disons tout de suite que l'espèce s'est révélée être une tique à trois hôtes, type de cycle habituel pour ce genre).

a) Période pré-ovipositaire :

9, 9, 12 et 14 jours (observations sur quatre femelles).

b) Période d'incubation :

$54,58,60$ et 61 jours (observations sur les œufs de quatre femelles).

c) Période de fixation des larves:

$1^{\circ}$ Sur lapin. Température et humidité ambiantes du laboratoire.

La période variait entre 6 et 8 jours (observations sur dix larves).

$2^{\circ}$ Sur Pyxis (deux spécimens).

La période variait entre 13 et 32 jours, avec une moyenne de 17 à 18 (observations sur 381 larves).

$3^{\circ}$ Sur Testudo radiata.

La période variait entre 16 et 20 jours (observations sur trois larves).

4. Sur Testudo planicauda.

La période variait entre 14 et 27 jours, avec une moyenne de 19 (observations sur 168 larves).

d) Période de mue des larves (entre le moment où les larves gorgées tombent et celui où el'es muent en nymphes) :

La période variait entre 17 et 26 jours, avec une moyenne d'environ 21 jours (observations sur 498 larves).

e) Période de fixation des nymphes:

$1^{\circ}$ Sur lapin. Température et humidité ambiantes, du laboratoire.

15 jours (observations sur deux nymphes).

$2^{\circ}$ Sur lapin. A l'étuve à tiques.

La période variait entre 7 et 10 jours (observations sur cinq nymphes). (Un incident fait s'arrêter cette observation au $10^{\circ}$ jour, alors que quelques nymphes étaient encore fixées).

$3^{\circ}$ Pyxis (trois spécimens).

La période variait entre 15 et 34 jours, avec une moyenne de 23 (observations sur 45 nymphes).

\section{$4^{\circ}$ Sur Testudo planicauda.}

La période variait entre 24 et 30 jours (observations sur trois nymphes).

f) Période de mue des nymphes:

La période variait entre 25 et 37 jours, avec une moyenne d'environ 30 (observations sur 46 nymphes). 


\section{g) Période de fixation des adultes:}

Sur Pyxis :

Cinq mâles et cinq femelles sont mis dans le cristallisoir. Trois femelles se gorgent et tombent en 28,29 et 31 jours; la tortue est alors trouvée morte ; deux femelles mi-gorgées et trois mâles sont encore fixés, les deux autres mâles se sont détachés, vivants.

\section{Influence de l'espèce de l'hôte.}

Les larves et les nymphes se sont gorgées bien plus rapidement sur lapin (animal à sang chaud) que sur les tortues (poïkilothermes). Il ne semble pas y avoir de différence notable entre les trois espèces de tortues quant à la durée de la période de fixation.

L'hôte a montré une influence marquée sur le pourcentage des larves et nymphes qui se gorgeaient et muaient par la suite :

Sur environ une centaine de larves mises sur lapin (climat du laboratoire), dix seulement se gorgent ; sept sur dix muent, les trois autres meurent avant la mue.

Sur environ une centaine de larves mises sur Testudo radiata, trois seulement se gorgent ; deux des trois larves sont laissées à l'étuve et muent toutes les deux.

Sur plusieurs centaines de larves (chiffre estimé de visu) mises sur Testudo planicauda, 168 se gorgent ; 164 sur 168 muent.

Sur un nombre semblable de larves mises sur Pyxis, 381 se gorgent ; 328 larves gorgées sont laissées à l'étuve ; 325 sur 328 muent.

Sur sept nymphes mises sur lapin (climat du laboratoire), la plupart se fixent, deux seulement se gorgent, mais meurent avant la mue.

Sur 37 nymphes mises sur lapin (climat de l'étuve), cinq se gorgent et quelquesunes sont encore fixées quand l'expérience est arrêtée (voir ci-dessus); trois sur cinq muent.

Sur 38 nymphes mises sur Testudo planicauda, trois seulement se gorgent ; toutes les trois muent.

Sur 129 nymphes mises sur Pyxis, 45 se gorgent (sur une Pyxis 27 sur 28, sur une autre 7 seulement sur 59 et sur une troisième 11 seulement sur 42 ). 40 sur 45 muent, cinq meurent avant la mue.

Trois mâles et trois femelles sont mis sur Testudo radiata; dans les quatre premiers jours, trois fois on a pu voir un mâle fixé, mais jamais plus de 24 heures; les jours suivants, aucune tique n'est fixée, et l'essai est abandonné après huit jours; toutes les tiques sont encore vivantes.

Sur cinq mâles et cinq femelles mis sur Pyxis, tous se fixent en moins de deux jours ; trois femelles se gorgent, les deux autres sont fixées, mi-gorgées, au moment de la mort de la tortue (voir ci-dessus), ainsi que trois des mâles, les deux autres mâles étant détachés.

Il ressort de ces observations que, parmi les animaux essayés, Pyxis arachnoides semble être l'hôte le plus convenable à $A$. chabaudi, mais qu'il n'est pas impossible, dans la nature, que quelques spécimens puissent se fixer et même se gorger sur d'autres 
espèces de tortue. A noter que, sur 15 Pyxis récoltées dans la nature, toutes étaient porteuses d' $A$. chabaudi, mais qu'aucune des 60 à 70 Testudo radiata, récoltées dans le même habitat, n'en portait (Malzy, 1964).

Ajoutons que les larves gorgées sur lapin et sur Testudo planicauda étaient presque toutes de couleur foncée, celles gorgées sur Pyxis étaient en général de couleur blanchâtre.

Lieu de fixation.

La plupart des larves et nymphes se fixaient sur les tortues au cou, sur les pattes et sur la queue ; de rares exemplaires s'attachaient sur la tête, sous le menton, et même



FIG. 1. - Pyxis arachnoides parasitée par deux femelles d'Amblyomma chabaudi

parfois dans une suture à l'extérieur du plastron, en parvenant à s'y gorger. Les quelques adultes placés avec Pyxis se fixaient sur les pattes et à la queue (voir figure 1). Malzy (1964) signale avoir récolté une femelle fixée dans une suture du plastron, et un mâle sur la carapace.

Proportion de mâles et de femelles après la mue des nymphes.

Au total, 24 mâles et 22 femelles ont été obtenus par l'élevage au laboratoire ; rappelons que M. Malzy a récolté dans la nature sur Pyxis, 24 mâles contre 4 femelles seulement, différence qui tient sans doute au fait que, comme il est connu pour d'autres espèces de tiques, les mâles n'ont pas de période déterminée de fixation, tandis que les femelles se laissent tomber quand elles sont gorgées. 


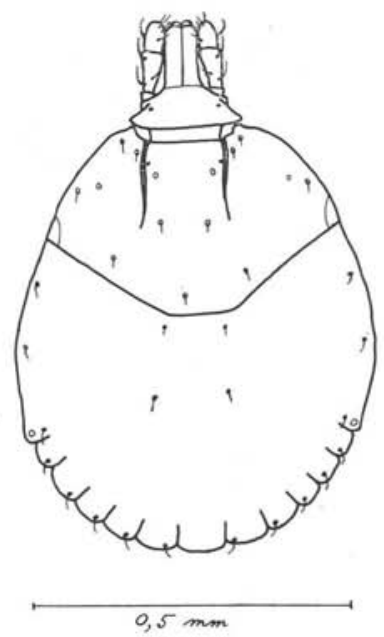

Fig. 2. - Amblyomma chabaudi, larve. Face dorsale.

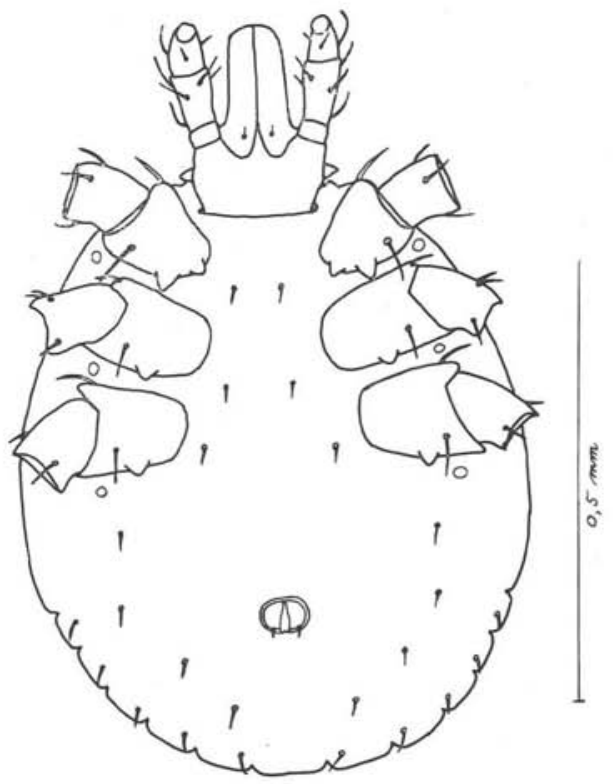

Fig. 3. - Amblyomma chabaudi, larve. Face ventrale. 
Description de la larve (fig. 2, 3 et 4)

\section{Dimensions :}

Larves à jeun :

Longueur (des pointes des scapulae à l'extrémité postérieure) $\times$ largeur: 0,58 à $0,66 \times 0,49$ à $0,59 \mathrm{~mm}$, en moyenne $0,64 \times 0.56$. (Mensuration sur 28 larves). (La longueur totale, rostre compris, était d'environ 0,74 à $0,83 \mathrm{~mm}$, moyenne 0,80 ; nous préférons toutefois mesurer les tiques des pointes des scapulae à l'extrémité postérieure, mesure plus précise, puisque le rostre est souvent infléchi ventralement).

Larves gorgées (sur Pyxis):

Longueur (des pointes des scapulae à l'extrémité postérieure) $\times$ largeur : 1,61 à $2,09 \times 1,21$ à $1,48 \mathrm{~mm}$, en moyenne $1,83 \times 1,36$. (Mensurations sur 14 larves ayant quitté spontanément l'hôte).

Face dorsale:

Scutum: Sans ornementation. Plus large que long: longueur $\times$ largeur de 0,273 à $0,297 \times 0,427$ à $0,460 \mathrm{~mm}$, en moyenne $0,284 \times 0,443$. (Longueur mesurée des pointes des scapulae jusqu'à l'extrémité postérieure de l'écusson). (Mensurations sur 15 larves à jeun). Bords antéro-latéraux convexes ; bords postéro-latéraux droits, ne se réunissant pas en un seul angle, mais aboutissant sur un court bord postérieur droit. Sillons cervicaux bien marqués, atteignant approximativement le niveau des yeux, parfois dépassant légèrement ce niveau; ils sont parallèles, puis, dans leur partie postérieure, légèrement divergents. Ponctuations et soies comme dessinées. Yeux plats.

Alloscutum: Pas de foveae visibles.

Capitulum : Basis capituli triangulaire. Bord postérieur droit, avec les extrémités latérales convexes. Angles latéraux aigus. Largeur (au niveau des angles latéraux) : 0,160 à $0,176 \mathrm{~mm}$, en moyenne 0,169 . (Mensurations sur 24 larves à jeun).

Rostre: Article III des palpes moins de deux fois plus long que l'article II.



$a$

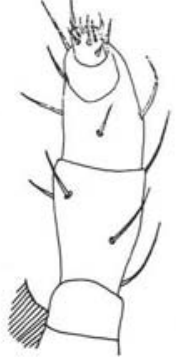

Fig. 4. - Amblyomma chabaudi, larve. a) Détails de l'hypostome et du palpe gauche en aspect ventral. b) Tarse I



$b$ 
Face ventrale :

Coxa I plus petite que coxae II et III. Coxa I avec deux éperons triangulaires, bien développés, l'éperon latéral presque deux fois plus long que l'éperon médial ; coxae II et III avec un éperon triangulaire, bien développé ; éperons des coxae II et III subégaux.

Hypostome : Dentition 2/2.

Soies comme desssinées.

\section{Observations complémentaires sur la nymphe}

\section{Dimensions :}

Nymphes à jeun :

Longueur (des pointes des scapulae à l'extrémité postérieure) $\times$ largeur : 1,35 à $1,85 \times 1,10$ à $1,53 \mathrm{~mm}$, en moyenne $1,66 \times 1,34$. (Mensurations sur 19 nymphes)

[Rappelons que la nymphe gorgée (Uilenberg, 1965) mesurait, sans rostre, $5,5 \times 4,0 \mathrm{~mm}]$.

Face dorsale :

Scutum: Plus large que long: 0,69 à $0,90 \times 0,85$ à $1,08 \mathrm{~mm}$, en moyenne (12 nymphes à jeun) $0,81 \times 0,99 \mathrm{~mm}$. (La nymphe gorgée décrite auparavant: $0,90 \times 1,03 \mathrm{~mm})$.

Bords postéro-latéraux droits à légèrement concaves (nymphes à jeun), angle postérieur arrondi.

Capitulum: largeur de la basis capituli: de 0,32 à $0,39 \mathrm{~mm}$, en moyenne (13 nymphes à jeun) $0,36 \mathrm{~mm}$. (La nymphe gorgée décrite auparavant: 0,37).

Alloscutum: Nous n'avions pas vu des foveae sur la première nymphe gorgée, sans doute par la distension du tégument. Mais, en réalité, la présence de deux foveae, comme sur les adultes, est constante sur les nymphes à jeun. Ces foveae se trouvent à une distance d'environ 0,20 à $0,25 \mathrm{~mm}$ derrière l'extrémité postérieure de l'écusson, une distance d'environ 0,15 à $0,16 \mathrm{~mm}$ les sépare entre elles, et leur diamètre n'excède pas environ $0,05 \mathrm{~mm}$; elles sont peu profondes. Un nouvel examen de la première nymphe gorgée n'a pas permis de discerner les foveae.

Voir aussi la description de la nymphe gorgée (Uilenberg, 1965).

\section{Femelles gorgées}

Les dimensions des trois femelles gorgées au laboratoire sur Pyxis étaient de: $16 \times 13,18 \times 15$ et $19,5 \times 15,5 \mathrm{~mm}$ (sans rostre).

\section{Identification à Madagascar}

La seule autre espèce du genre trouvée jusqu'ici dans le pays est Amblyomma variegatum (Fabricius, 1794). [Il est vrai que Neumann (1901) signale la présence d'une autre espèce, A. rhinocerotis (De Geer, 1778) (sous le nom de A. petersi Karsch, 
1878, probablement synonyme d'après Hoogstraal, 1956), mais il s'agit vraisemblablement d'une erreur (Hoogstraal, 1953). Quant à Amblyomma hebraeum Koch, 1844, l'espèce a été trouvée à plusieurs reprises sur des animaux importés à Tamatave, mais elle n'a pas réussi à s'établir dans le pays (Bück, 1948, Uilenberg, 1964)].

Les différences entre les adultes d' $A$. chabaudi et d' $A$. variegatum sont si nombreuses et frappantes, qu'il n'est pas nécessaire de s'y attarder.

Quant aux nymphes, elles aussi se distinguent facilement; quelques différences frappantes :

Yeux : plats chez $A$. chabaudi, hémisphériques chez A. variegatum.

Coxae: éperons des coxae II, III et IV subégaux chez $A$. chabaudi, tandis que les éperons des coxae II et III sont plus courts que celui de coxa IV chez $A$. variegatum. Coxa I a deux éperons de longueur subégale chez $A$. chabaudi, l'éperon latéral est nettement plus long que le médial chez $A$. variegatum.

Les larves diffèrent également de façon nette de celles d' $A$. variegatum; quelques différences faciles à constater :

Yeux : plats chez $A$. chabaudi, bombés chez $A$. variegatum.

Coxae : éperons nets, triangulaires chez $A$. chabaudi, très courts et arrondis chez A. variegatum.

Les dimensions sont également différentes, mais elles peuvent se chevaucher légèrement, et ne donneront donc pas toujours une indication sûre: longueur $\times$ largeur de l'écusson chez $A$. chabaudi (voir ci-dessus) : 0,27 à $0,30 \times 0,43$ à $0,46 \mathrm{~mm}$, chez $A$. variegatum: 0,29 à $0,33 \times 0,49$ à $0,53 \mathrm{~mm}$ (mensurations sur 12 larves à jeun d'A. variegatum) ; les moyennes respectives sont : $0,28 \times 0,44$ et $0,32 \times 0,51 \mathrm{~mm}$. (Les yeux saillants chez $A$. variegatum sont compris dans la largeur).

Largeur de la basis capituli : chez $A$. chabaudi 0,16 à $0,18 \mathrm{~mm}$ (voir ci-dessus), chez $A$. variegatum 0,18 à $0,19 \mathrm{~mm}$ (mensurations sur 15 larves à jeun); les moyennes respectives sont : 0,17 à $0,19 \mathrm{~mm}$.

Remerciements : Nous remercions M. P. Malzy de nous avoir fourni la femelle qui est à l'origine de l'élevage ; nous lui sommes aussi très reconnaissant d'avoir bien voulu nous prêter les tortues.

\section{Résumé}

L'auteur rapporte les résultats de l'élevage au laboratoire des différents stades de la tique Amblyomma chabaudi Rageau, 1964, sur les tortues Pyxis arachnoides, Testudo radiata et $T$. planicauda, ainsi que sur lapin. La durée du cycle (qui est celui d'une tique à trois hôtes) est très longue sur les tortues poïkilothermes. $P$. arachnoides semble mieux convenir à cette tique que les autres tortues et le lapin. La larve est décrite et illustrée, et la description de la nymphe est complétée. Quelques différences avec les nymphes et les larves d' $A$. variegatum, seule autre espèce du genre à Madagascar, sont indiquées. 


\section{Summary}

The results are given of breeding the different stages of the tick Amblyomma chabaudi Rageau, 1964, on the tortoises Pyxis arachnoides, Testudo radiata and $T$. planicauda, as well as on rabbits. Duration of the cycle (which is of the three host type) is very long on the cold-blooded tortoises. $P$. arachnoides seems a better host than the other tortoises and the rabbit. The larva is described and illustrated, and the description of the nymph is completed. Some of the differences with the nymphs and larvae of A. variegatum, only other species of the genus in Madagascar, are indicated.

\section{Bibliographie}

BaILEY K. P., 1960. - Notes on the rearing of Rhipicephalus appendiculatus and their infection with Theileria parva for experimental transmission. Bull. epiz. Dis. Afr., 8: 33-41.

Buck G., 1948. - Tiques des animaux domestiques à Madagascar. Bull. agricole, Madagascar, $1: 3-11$.

Hoogstraal H., 1953. - Ticks (Ixodoidea) of the Malagasy faunal region (excepting the Seychelles). Their origins and host-relation-ships; with descriptions of five new Haemaphysalis species. Bull. Mus. comp. Zool. Harvard, 111: 37-113.

-, 1956. - African Ixodoidea. Volume I. Ticks of the Sudan. U.S. Dept. of the Navy, Bureau of Medicine and Surgery, Research Report NM 005 050-29-07.

Malzy P., 1964. - Sur Pyxis arachnoides Bell, tortue terrestre du Sud de Madagascar. Bull. Mus. Nat. Hist. Nat., $2^{\circ}$ Série, $36: 441-443$.

Neumann G., 1901. - Révision de la famille des Ixodidés. (4eme Mémoire). Mem. Soc. Zool. France, 14: 249-372.

Rageau J., 1964. - Une nouvelle espèce d'Amblyomma parasite de tortues malgaches $A$. Chabaudi n. sp. (Acariens-Ixodidae). Bull. Soc. Path. exot., 57: 408-411.

Uilenberg G., 1964. - Notes sur les hématozoaires et tiques des animaux domestiques à Madagascar. Rev. Elev. Méd. vét. Pays trop., 17: 337-359.

-, 1965. - Amblyomma chabaudi Rageau, 1964. (Ixodidae). Description de la femelle et de la nymphe. Variations morphologiques du mâle. Ann. Parasit. hum. comp., $40: 681-691$.

[Institut d'Elevage et de Médecine Vétérinaire des Pays tropicaux. - Laboratoire Central de l'Elevage. - Service d'Entomologie-Protozoologie, Tananarive (Madagascar)] 